\title{
Correspondence
}

\section{Senior emergency department staff also need mental health training}

As a junior doctor who has moved to psychiatry after previously spending 2 years training in emergency medicine, I read Dr Gordon's article with interest. ${ }^{1}$ The survey clearly highlighted a need for mental health training of new doctors working in the emergency department. During one of my training posts in emergency medicine, I completed an audit which showed that $75 \%$ of patients presenting to the department with self-harm were being seen by junior medical staff and highlighted the need for increased training and supervision of junior emergency department doctors. In addition, many such patients present out of hours when access to senior support and psychiatric services may be more limited.

I think that it is also important to consider the knowledge, skills and attitudes of senior doctors working in the emergency department. Assessment and treatment of the patient presenting with self-harm and behavioural disturbance does form part of the College of Emergency Medicine curriculum. However, in my experience, many senior doctors working in emergency medicine - with some exceptions - have little interest in assessing and treating patients who present with self-harm or other mental health problems. As my audit showed, many such patients are left to junior doctors to see. When asked for advice about such patients, a common response from senior doctors is to advise that the patient should be referred to 'psych', without any meaningful discussion or assessment of the patient; this is in contrast to patients presenting with other problems such as trauma or minor injuries, when a senior doctor may show more interest in seeing the patient and teaching their junior staff.

It would be beneficial for patients if links between emergency departments and psychiatric liaison services were improved and if increasing the amount of mental health training available for all grades of doctors working in emergency departments was considered.

1 Gordon JT. Emergency department junior medical staff's knowledge, skills and confidence with psychiatric patients: a survey. Psychiatrist 2012; 36: 186-8.

Thomas J. Burden, CT1 in general adult psychiatry, Links Community Mental Health Team, Cardiff, UK, email: thomasburden@doctors.org.uk doi: $10.1192 /$ pb.36.8.314

\section{Simulation training: a tool to improve junior doctors' confidence}

We read Dr Gordon's paper ${ }^{1}$ with great interest as it echoes our own work while surveying the confidence of junior doctors new to psychiatry. Like Dr Gordon, we felt there was a specific need to combat their self-perceived lack of confidence, particularly in the out-of-hours environment, as often more senior supervision is based off site. However, we do feel that Dr Gordon's recommendations for 'mental health training of new doctors working in the emergency department' should perhaps be expanded to all those new to psychiatry, as the identified difficulties are not unique to the liaison service.
This issue has potential effects on patient safety, which is substantiated by a third of foundation year 1 respondents in the British Medical Association's study reporting that they had been asked 'to undertake tasks which they felt were beyond their capabilities' during their placements. ${ }^{2}$

In our own work, we expressly aimed to target this situation, consequently designing and implementing a simulation-based programme as part of the induction process of our trust. Simulation-based training has been recommended as a risk-free and efficient way of improving the quality of junior doctors' training. ${ }^{3}$ Junior doctors new to psychiatry participated in a range of complex, clinical, out-of-hours simulated scenarios under the observation of experienced consultants and patient representatives. Timely focused feedback was given by the observers, and the doctors had an opportunity to discuss their performance within clinical supervision sessions using recorded video. We received positive feedback from the participants, including a selfreported increase in their confidence when this was measured in follow-up sessions. They also felt that this would have an impact on their performance with real patients. The patient representatives gave a unique viewpoint and they felt that there were clear improvements in trainees' performance following the first session.

We recently presented our small-scale pilot in a London Deanery 'Quality and innovation' conference, where it was well received. It generated interest from other trusts that were keen to potentially implement similar programmes locally.

We ultimately hope that our project will be used to increase and focus supervision in out-of-hours work, while also improving patient safety using engaging and interactive learning through simulation.

1 Gordon JT. Emergency department junior medical staff's knowledge, skills and confidence with psychiatric patients: a survey. Psychiatrist 2012; 36: 186-8.

2 British Medical Association. Cohort Study 2006: Medical Graduates (Third Report). BMA, 2009.

3 Temple J. Time for Training: A Review of the Impact of the European Working Time Directive on the Quality of Training. Medical Education England, 2010.

Rhodri S. David, Fellow in Medical Education and Honorary Clinical Lecturer, Mohan Bhat, Director of Medical Education and Honorary Senior Lecturer, North East London NHS Foundation Trust, Goodmayes, Ilford, email: rhodri.david1@nhs.net

doi: 10.1192/pb.36.8.314a

\section{CASC candidates need better preparation}

Kashyap \& Sule are right to express outrage at the low pass rates for the Royal College of Psychiatrists Clinical Assessment of Skills and Competencies (CASC), and concern over the difference between UK-trained candidates and those trained elsewhere. ${ }^{1}$ They offer good suggestions for improvement. However, by focusing on the examination itself rather than the quality of CASC preparation in UK postgraduate training programmes, their outrage may be misdirected. 
The validity and reliability of multistation Objective Structured Clinical Examinations (OSCEs) has been tentatively established. ${ }^{2}$ Despite the appeal of the long-case examination, it has poor interrater and test-retest reliability when systematically evaluated; ${ }^{3}$ its continued use in high-stakes professional examinations is difficult to justify. However, it is very concerning that many candidates are surprised when failing a supposedly objective examination after 3 years of practising psychiatry. Can it be that so many intelligent and diligent psychiatry residents have a severe lack of insight into their own abilities? This seems implausible. It is more likely that postgraduate training programmes are failing to equip residents with the skills they need to pass the CASC. Given that these are predominantly consultation and interpersonal skills, it is difficult to escape the conclusion that residents receive inadequate feedback on clinical skills in their initial years of practice, even before commencing formal preparation for the CASC.

The College dropping the Part 1 OSCE shifted responsibility for evaluating first-year residents' core clinical skills to postgraduate training programmes by means of the workplacebased assessment (WPBA) system. This approach is not effective: there are multiple flaws in the current WPBA system ${ }^{4}$ and its suitability for assessing and developing core clinical skills is even more questionable than the long-case examination. ${ }^{5}$ These observations are supported by our own experience of delivering CASC training: many candidates are surprised to receive in-depth feedback on difficulties in interpersonal and consultation style. After 3 years of practising psychiatry to their best of their ability with little criticism or coaching, it is no wonder that they are disappointed when the first piece of negative feedback they receive is failing the CASC. This affects UK-trained and non-UK-trained candidates alike and to focus on discrepancies detracts from the issue that the current pass rate is too low for all candidates.

This leads us to the conclusion that a substantial share of responsibility for low CASC pass rates lies not with the Royal College of Psychiatrists, but with the postgraduate training programmes. It is of course important that the CASC is continuously evaluated and improved, but there are more pressing issues. First, we suggest that training programme directors collect and publish data on CASC pass rates and urgently improve support and training for residents at risk of failing. Second, preparation for the CASC must start in the first year of psychiatric practice, in the form of in-depth consultation skills training beyond the WPBA system. Finally, we recommend that current and prospective psychiatry residents use all available information regarding the quality of clinical skills and CASC training when choosing a postgraduate training programme.

\section{Declaration of interest}

A.T. and D.H. deliver CASC revision training although do not profit from it. D.H. is author of Deconstructing the OSCE, due to be published in 2013 by Oxford University Press.

1 Kashyap G, Sule A. MRCPsych CASC exam: is there a better choice? Psychiatrist 2012; 36: 197.

2 Hodges BD, Regehr G, Hanson M. Validation of an objective structured clinical examination in psychiatry. Acad Med 1998; 73: 910-2.

3 Leichner P, Sisler GC, Harper D. A study of the reliability of the clinical oral examination in psychiatry. Can J Psychiatry 1984; 29: 394-7.
4 Menon S, Winston M, Sullivan G. Workplace-based assessment: attitudes and perceptions among consultant trainers and comparison with those of trainees. Psychiatrist 2012; 36: 16-24.

5 Kahn MJ, Merrill WW, Anderson DS, Szerlip HM. Residency program director evaluations do not correlate with performance on a required 4th-year objective structured clinical examination. Teach Learn Med 2001; 13: 9-12.

Alex B. Thomson, locum consultant liaison psychiatrist, Central and North West London NHS Foundation Trust, email: alex.thomson@nhs.net, Duncan Harding, NIHR Clinical Lecturer, Department of Forensic and Neurodevelopmental Sciences, Institute of Psychiatry, King's College London

doi: $10.1192 / \mathrm{pb} .36 .8 .314 \mathrm{~b}$

\section{The need for age-appropriate forensic services}

Dr Connolly has rightly pointed out that planning for the development of mental health services requires an understanding of the changing demographics in the country. ${ }^{1}$ We feel every directorate within psychiatry will need to prepare for the demographic transition through thoughtful planning in service development that can provide quality as well as appropriate care to the elderly. Within forensic services, serious thought should be given to development of geriatric forensic service.

Traditionally, it is believed that there is a low crime rate in the elderly. However, studies have shown that there is an increase in criminal behaviour among those 60 or over, $^{2}$ and the number of people in prison over the age of 60 has grown from 1.3 to $2.4 \%$ in England. ${ }^{3}$

A study by Needham-Bennett et al concluded that there is a high prevalence (28\%) of psychiatric disorders in alleged offenders in the community aged 60 years and over. ${ }^{4}$ Studies done in the prison populations have shown that the prevalence of psychiatric disorders among remanded male prisoners aged 55 years and over was $50 \%{ }^{5}$ and in sentenced male prisoners $53 \% .^{2}$

Moreover, up to a half of elderly offenders with psychiatric disorders have a physical illness. In addition, they may have visual impairment, auditory impairment, mobility problems and cognitive impairment. Currently, forensic mental health units with long-term rehabilitation wards provide care for elderly individuals. This longer-term admission is usually due to ongoing risks combined with difficulties in rehabilitating this patient group because of 'institutionalism' or ongoing mental health issues. We wondered whether such units were equipped to be able to deliver care for older individuals with increasing physical comorbidities or those who develop certain organic conditions such as dementia. Another issue that needs consideration is the use of risk assessment tools such as Historical Clinical Risk Management 20 (HCR-20) in the older age group in forensic units that are generally used for workingage individuals. It is our view that the current psychological treatment programmes such as the sexual offending treatment programme will need modifications for this client group.

We feel that the complex needs of elderly mentally disordered offenders appear to fall within the domains of geriatric psychiatry services and forensic psychiatry services, but they may not be met by either service alone. Consideration should be given to setting up specialist tertiary forensic geriatric psychiatry. There has been some initiative in the independent sector in this matter.

1 Connolly M. Futurology and mental health services: are we ready for the demographic transition? Psychiatrist 2012; 36: 161-4. 